\title{
In-vivo evaluation of implantable film containing microencapsulated sustained release dexamethasone sodium phosphate
}

\author{
Mohammed Wajeah Abdul Ameer, ${ }^{a}$ Nidhal K. Maraie, ${ }^{a}$ Ali Mohsin Alwaelib
}

\author{
aDepartment of Pharmaceutics, College of Pharmacy, Mustansiriyah University, Baghdad, Iraq \\ 'Department of Human Anatomy, College of Medicine, Mustansiriyah University, Baghdad, Iraq \\ Corresponding author: Nidhal K. Maraie (Email: pharma.dr.nidhal.khazaal@uomustansiriyah.edu.iq) \\ ORCID: 0000-0001-5628-1479 \\ (Submitted: 19 September 2019 - Revised version received: 03 October 2019 - Accepted: 21 October 2019 - Published online: 26 December 2019)
}

\begin{abstract}
Objective This work involved in vivo evaluation of the previously prepared implantable film dosage form containing microencapsulated dexamethasone sodium phosphate (which is equivalent to $16 \mathrm{mg}$ dexamethasone) and $4.4 \mathrm{mg}$ pure dexamethasone sodium phosphate (which is equivalent to $4 \mathrm{mg}$ dexamethasone) to be inserted at the inflamed areas to get suitable precise controlled release.

Methods The animals used were 33 rats divided into 8 groups and the inflammation was induced by using $2.0 \%$ carrageenan solution to be injected intradorsally. Then the film was inserted locally and then sacrifying the animals on different time tables to take sample from the site of inflammation for 5 months and examined under electron microscope

Results The results showed that the prepared implantable film had immediate anti-inflammatory effect upon insertion at the inflamed tissue and its action continued effectively for 5 months.

Conclusion This work proved that implantable film containing dexamethasone should be used after surgery of joint replacement or any other surgery site as alternative to the applied regimen of giving intramuscular injection of the commercially available 10 mg dexamethasone intraoperatively and a second $10 \mathrm{mg}$ within 24-hour post-operatively to reduce edema and pain followed by oral dexamethasone $0.5 \mathrm{mg}$ daily till complete recovery which may last for 5 months or more and subject the patients to the serious systemic effects. The application of the implanted film therapy may reduce the rejection of the body to the replaced joint or other replacement surgery and improves patient compliance.

Key words microencapsulated, dexamethasone sodium phosphate, carrageenan solution
\end{abstract}

\section{Introduction}

Inflammation is evolved as a beneficial strategy for the host in response to any potential danger, the inflammatory response is normally terminated once the potential danger is eradicated. Usually the reversion of the inflammatory response to the homeostatic state precedes quite rapidly as a highly regulated process known as the resolution of inflammation. ${ }^{1}$ If the resolution of inflammation fails for any reason, the acute inflammation turns into a chronic stage. A chronic inflammation is generally believed to develop if the elimination of the triggering stimulus fails to happen, such as any persistent infection or chronic cellular injury. ${ }^{2}$

Biomaterials are engineered to take a form that can work alone or as part of a complex system providing direction to the course of any therapeutic procedure by regulating interactions with components of living systems ${ }^{3}$ Following the implantation of biomaterials in vivo, host reactions incorporate a combination of many processes including, blood-material interactions, provisional matrix formation, inflammation (acute then chronic), development of granulation tissue, foreign body reaction, and fibrous capsule development. ${ }^{4}$

Blood-biomaterial interactions begin to occur concurrently after bio-implantation, with protein adsorption to the biomaterial surface and the development of a blood-based transient provisional matrix (initial thrombus at the tissue/ material interface) that forms on and around the biomaterial. ${ }^{5}$ The provisional matrix is rich in cytokines, growth factors, and chemo-attractants that are capable of recruiting cells of the innate immune system to the injury site. ${ }^{6}$ Following this provisional matrix formation, acute inflammation, and subsequently, chronic inflammation occur sequentially. The degree of these responses is dependent on the extent of injury during the implantation procedure.

An example of implantation is joint replacement. Several million people worldwide each year undergo a total joint arthroplasty-the prosthetic replacement of these destroyed native weight-bearing surfaces, ${ }^{7} 10-15 \%$ of these joint replacements will fail, and the single most common cause of failure, and reason for revision, is a process known as osteolysis. ${ }^{8}$ Debris forming from the wear of the prosthetic implants is responsible for the initiation of this aseptic inflammatory response. Osteolysis incidence rates have generally been reported to lie in the range of 5-20\%, but have been as high as $40 \%$ in some studies. ${ }^{9}$ Incidence rates are also predicted to substantially increase over time, in light of the fact that $35-45 \%$ of patients undergoing total joint replacement are now under the age of 65 years.

High-dose systemic steroid plays a critical role in rapid recovery of the patient after joint replacement and also decrease nausea, vomiting, and pain post-operatively. ${ }^{10}$ However, systemic side effect is the major limitation for the long-term use of steroids. ${ }^{11}$ One way to decrease such side effect is through using local delivery system such as implantable film in which the drug release will be locally on the site of implantation.

Dexamethasone sodium phosphate (DSP) is a sodium phosphate salt form of dexamethasone, a synthetic adrenal corticosteroid with potent anti-inflammatory properties. It is used to reduce edema and pain after joint replacement or any other surgery when given in the form of IM injection of the commercially available $10 \mathrm{mg}$ dexamethasone intraoperatively and a second $10 \mathrm{mg}$ within 24 -hour post-operatively to followed by oral dexamethasone $0.5 \mathrm{mg}$ daily till complete recovery which may last for 5 months or more. ${ }^{12}$ 
The aim of the work is to evaluate (in vivo) a thin film containing microencapsulated dexamethasone (previously prepared in our lab.) to be implanted at the inflamed to be used as alternative to dexamethasone conventional regimen treatment as anti-inflammatory to reduce invasive administration and frequent doses leading to reducing side effect and improves patients compliance, and correlate the in vitro results to in vivo response.

\section{Materials and Methods}

\section{Materials}

Aluminum chloride and anhydrous calcium chloride were purchased from Laboratory chemical, India. Benzalkonium chloride was purchased from Sinopharm Chemical reagent, China. Potassium phosphate monobasic was purchased from Himeda-India. Sodium azide was purchased from Panreac AAG, Spain. Carrageenan was purchased from Baoji guokang, China

\section{Content of the Prepared Implantable Film}

The prepared film contains sustained release DSP microcapsules containing $17.6 \mathrm{mg}$ DSP (which is equivalent to 16 mg dexamethasone) in which poly(lactic-co-glycolic acid) (PLGA) was used as a coating material and $4.4 \mathrm{mg}$ pure DSP (which is equivalent to $4 \mathrm{mg}$ dexamethasone). A thin film (2 $\mathrm{cm} \times 0.2 \mathrm{~cm}$ ) was obtained. ${ }^{13,14}$

\section{Preparation of Carrageenan Solution:}

The solution was prepared by adding $500 \mathrm{ml}$ of sterile $0.9 \%$ saline into a 1-L beaker with stirring using magnetic stirrer. Then slowly add $10.0 \mathrm{~g}$ carrageenan powder to the beaker and heat the solution to $90^{\circ} \mathrm{C}$ with stirring, without allowing the solution to boil. Heating the mixture helps to dissolve carrageenan; it took less than an hour to dissolve all of the powder. The obtained solution $(2.0 \%)$ was poured into a clean $1-\mathrm{L}$ glass bottle equipped with a cap and sterilize by autoclaving for $30 \mathrm{~min}$. Cool the solution to room temperature and aliquot into sterile, 100 -ml capped bottles and inject $100 \mu \mathrm{l}$ subcutaneously to induce inflammation in the rats. ${ }^{15}$ The effect of one subcutaneous injection of carrageenan solution stays for 1 week. ${ }^{16}$

\section{Animals Used}

This study was performed on Norway Albino rats. Animals were kept in the animal house and according to the ethics, in the College of Pharmacy, Mustansiriyah University, under natural light-dark cycles \& environmental temperature of $23-25^{\circ} \mathrm{C}$. Animals had free access to tap water \& pellet food. Accepted standards of animal care were used.

\section{Grouping of the Animals}

The total number of animals used was 33 rats which were divided into 8 groups as shown in Table 1.

- The animals used in group 1 were not treated with any agents and were used to see the shape of the normal tissue.

- The animals in group 2 were treated with inflammatory inducing agent (as SC injection) carrageenan and sacrificed after 1 day to see the infiltration of inflammation cell to the tissue (sign of inflammation).

- The animals used in group 3 were injected with SC carrageenan solution weekly for 1 month, then the animals were sacrificed at the end of the month to see the sign of inflammation occur at the end of this period. ${ }^{15}$

- The animals in group 4 were injected with carrageenan solution (SC) and then insert an empty (placebo) implantable film (i.e.. film contain only PVA (polyvinyl pyrrolidine)) base and did not contain drug in the dorsal neck area and then sacrificed the animals after 1 day to study the sign of inflammation and also to compare the inflammation happened with that result from group 2 .

- The above groups (from group 1 to 4 ) were considered as control groups

- The animals in group 5 were injected with carrageenan solution (SC) and injected with DSP solution at a dose of $2.25 \mathrm{mg} / \mathrm{kg}$ as IM injection at day 0 , and then sacrificed the animals after 1 day to study the immediate effect of injection DSP as IM on the treatment of the inflammation created by carrageenan injection. ${ }^{14}$

- The animals in group 6 were injected with carrageenan solution (SC) on weekly base to induce inflammation and injected IM DSP solution at day 0 with a dose of $2.25 \mathrm{mg} /$ $\mathrm{kg}$ and then sacrificed the animals at the end of the month to study the effect of injection DSP IM on long-term effect.

- The animals in group 7 were injected with carrageenan solution (SC) to induce inflammation and insert DSP implanted film at the site of injected carrageenan solution

Table 1. The groups of animals used during in-vivo evaluation of DSP implantable film

\begin{tabular}{llc}
\hline No of group & \multicolumn{1}{c}{ The name of the group } & No of animals in the group \\
\hline Group 1 & Control group & 2 \\
Group 2 & Carrageenan after 1 day & 2 \\
Group 3 & Carrageenan after 1 month & 2 \\
Group 4 & Carrageenan with empty disc after 1 day & 2 \\
Group 5 & Carrageenan with IM injection of dexamethasone after 1 day & 5 \\
Group 6 & Carrageenan with IM injection of dexamethasone after 1 month & 5 \\
Group 7 & Carrageenan with implantable film of dexamethasone sodium phosphate after 1 day & 5 \\
Group 8 & Carrageenan with implantable film of dexamethasone sodium phosphate after & 10 \\
\hline
\end{tabular}


and then sacrificed the animals after 1 day to study the effect of implantable DSP on the inflammation sign after 1 day.

- The animals in group 8 were injected with carrageenan solution (SC) every week for a month to induce inflammation and insert single DSP film at the site of injection of carrageenan solution (at zero time), and then sacrificed 2 animals from the group after 1 month to study the effect of implantable DSP on the inflammation sign after 1month. The same work was repeated for 5 months by scarifying 2 animals per month.

\section{Sampling}

The rats were first sacrificed by putting them in a closed jar containing vaporized chloroform for 2-3 min. Then incision with $5 \mathrm{~cm}$ length was made at the site of injectable carrageenan solution (i.e., dorsal neck) and 3 types of sample were taken from the skin, subcutaneous area, and muscle to study the type and number of infiltration of inflammatory cell in these tissue. The samples were stained with hematoxylin \& eosin to be examined under light microscope. ${ }^{16}$ Photomicrographs were taken as snap-shots using the camera and included all regions of the slides in different magnifications (10X and 40X).

\section{Result and Discussions}

Fig. 1 showed the condition of skin, subcutaneous tissue, and muscle tissue for normal rats (group 1) and for inflamed cells (group 2, $24 \mathrm{~h}$ after carrageenan injection), where group 2 cells showed marked increase in the infiltration of inflamed cells in the subcutaneous and muscle tissues specially neutrophils,

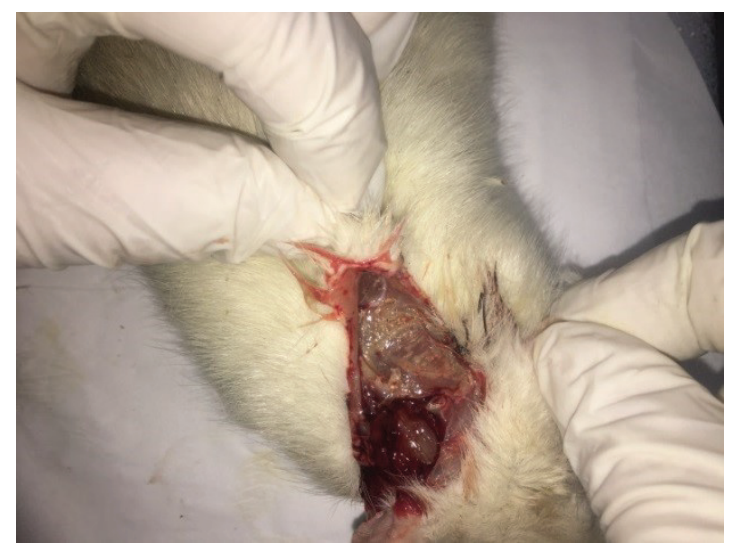

Fig. 2 Visual inspection of the injected area (group 2) after SC carrageenan injection.

which is usually increased during acute inflammation while no such sign was observed in skin tissue. ${ }^{17}$

The visual inspection of the injected area (in group 2) showed an excretion of yellow exudate after opening it with a marked edema as shown in Fig. 2.

In group 3, the animals (after 1 month from weekly SC injection of carrageenan solution) showed no significant increase in the infiltrations of the inflamed cells in subcutaneous and muscle tissue (while skin tissue stayed with no sign of inflammation) in comparison to rats of group 2, since carrageenan induced its maximum effect after $24 \mathrm{~h}$ from single injection and its effect maintained for 1 week. ${ }^{18}$ Therefore, single injection of carrageenan is enough to maintain acute inflammation for 1 week. However, it was found that after every week injection for 1 month (Fig. 3), most of the inflamed

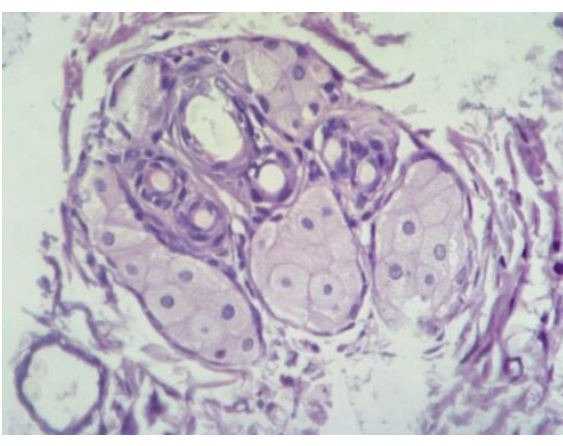

A) Skin

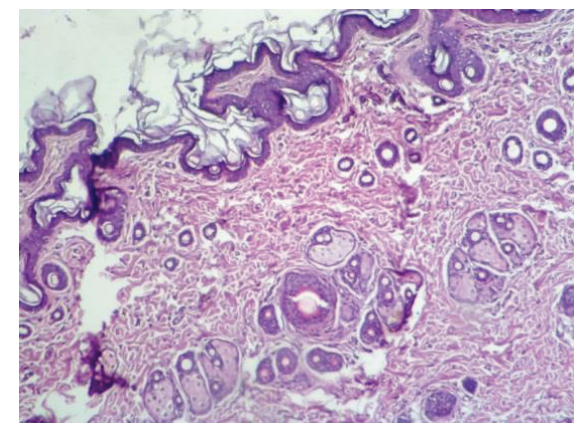

B) Skin

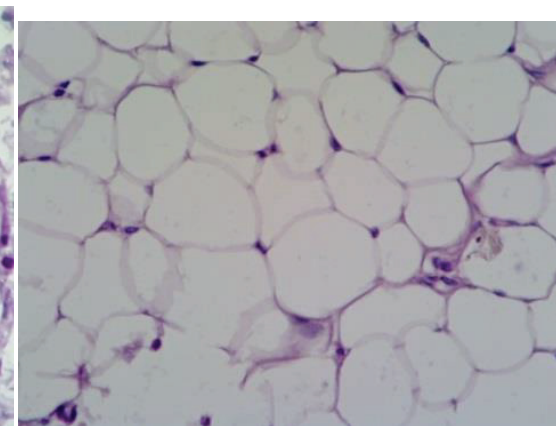

Subcutaneous tissue

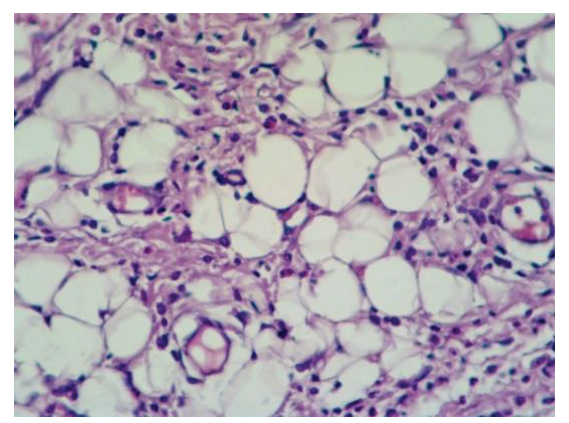

Subcutaneous tissue

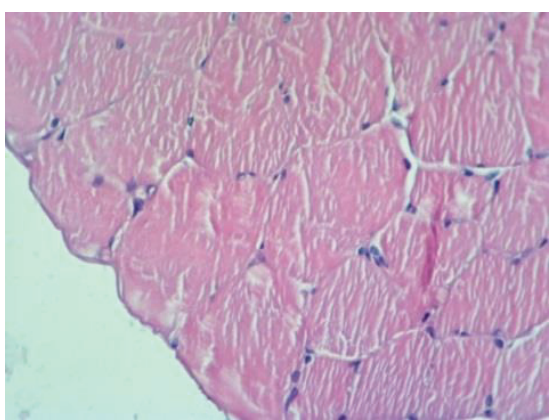

Muscle tissue

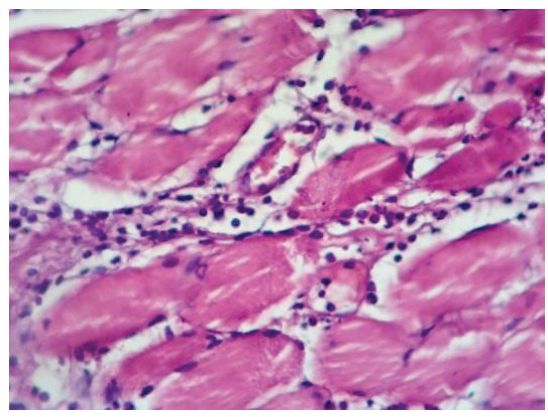

Muscle tissue

Fig. 1 Skin, subcutaneous and muscle tissue condition for (A) normal rats in group 1, (B) inflamed rats in group 2. 


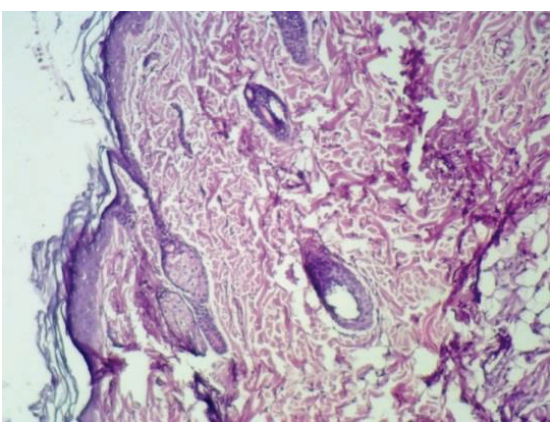

Skin

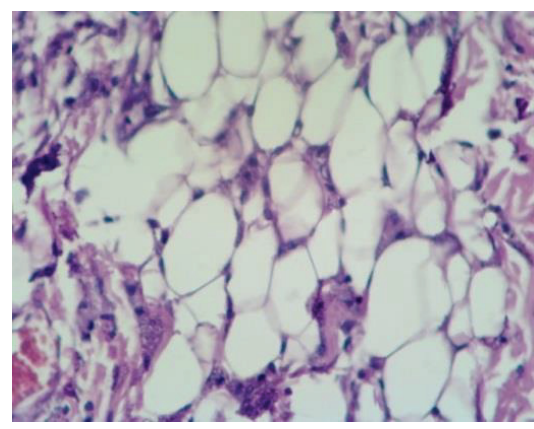

Subcutaneous

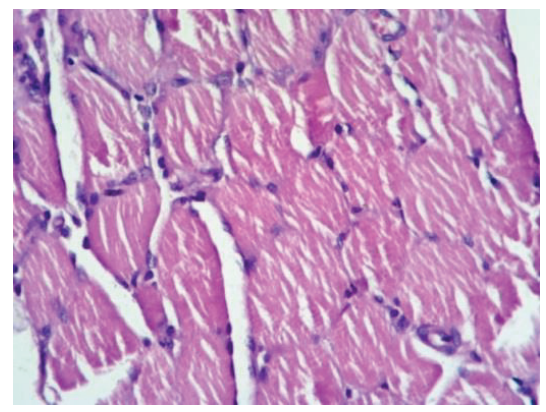

Muscle

\section{Fig. 3 Skin, Subcutaneous and muscle tissue condition after 1 month from weekly SC injection of carrageenan solution.}

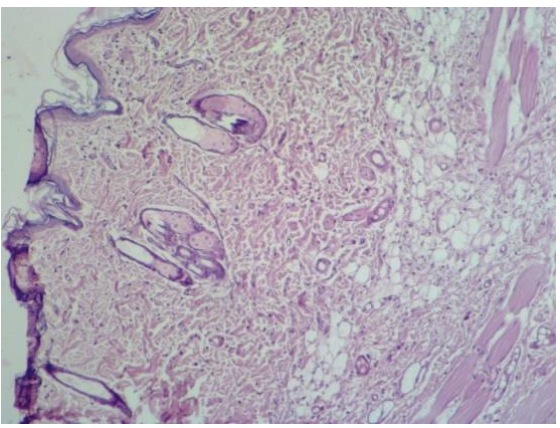

Skin

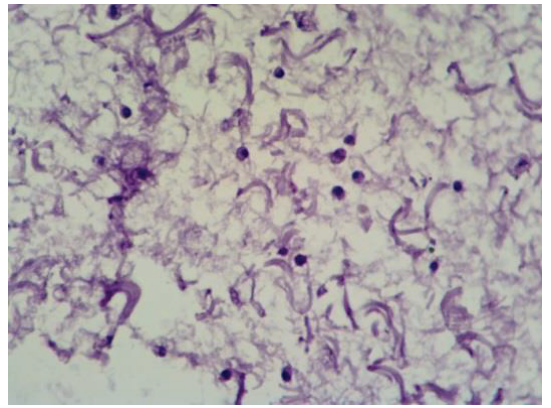

subcutaneous

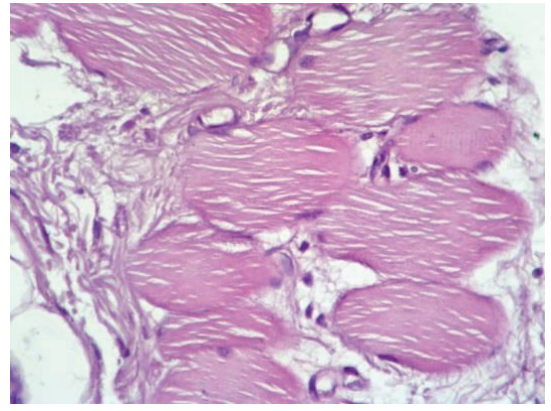

muscle

Fig. 4 Skin, subcutaneous, and muscle tissue conditions for inflamed cells after 1 day of single SC injection of carrageenan solution and insertion of placebo film at the same time.

cells found at the inflammation site showed an increase in the lymphocyte cells indicating chronic inflammation ${ }^{19}$ (while main cells in rats of group 2 were neutrophils indicating acute inflammation). Therefore, single injection of carrageenan solution was adapted to induce acute inflammation (for 1 day and stayed for a week), while every week injection for 1 month and more was adapted to induce chronic inflammation.

The samples taken from group 4 in which the animals in this group injected with carrageenan solution (SC), at the same time a placebo implantable film was inserted at the site of carrageenan injection and the condition of the tissue was followed up after $24 \mathrm{~h}$. There was a non-significant increase in the infiltration of inflamed cell in the subcutaneous and muscle tissue while there was no sign of inflammation at the skin tissue (Fig. 4 ) in comparison to group 2, which indicated that the base and additives of the implanted film had no effect on tissue condition and the inflammation is attributed to carrageenan only and the film was completely dissolved within 30 $\min$.

The samples taken after 1 day from group 5 in which the animals in this group injected with SC injection of carrageenan and intramuscular injection of DSP solution (at the same time) showed no sign of inflammation in the skin tissue, marked decrease in the infiltration of inflamed cell in the muscle tissue, and some inflamed cells in subcutaneous tissue in comparison to group 2 (which was injected with SC carrageenan alone) as shown in Fig. 5. This indicated that the DSP has an immediate, good, and potent anti-inflammatory effect

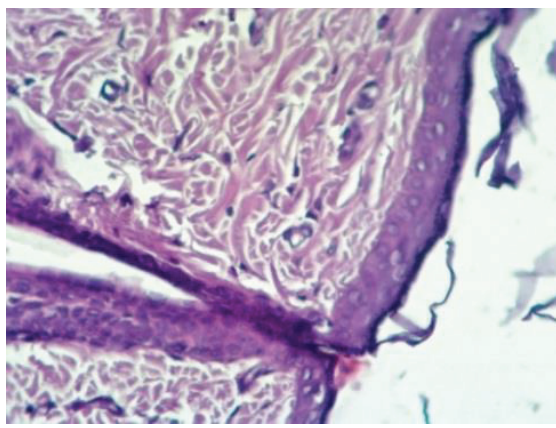

Skin

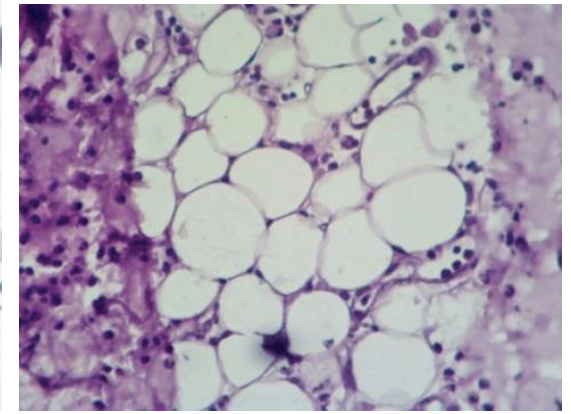

Subcutaneous

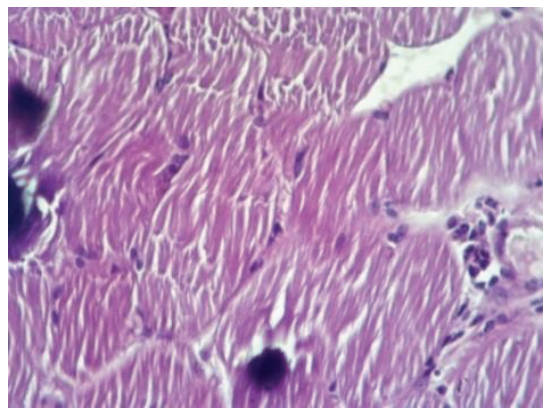

Muscle

Fig. 5 Skin, subcutaneous, and muscle tissues condition after 1 day of single SC carrageenan injection and IM injection of DSP solution. 


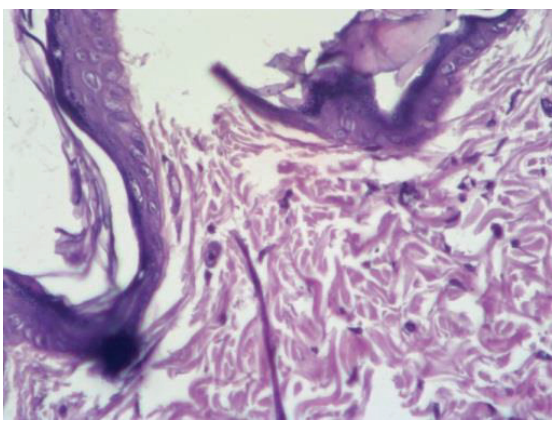

Skin

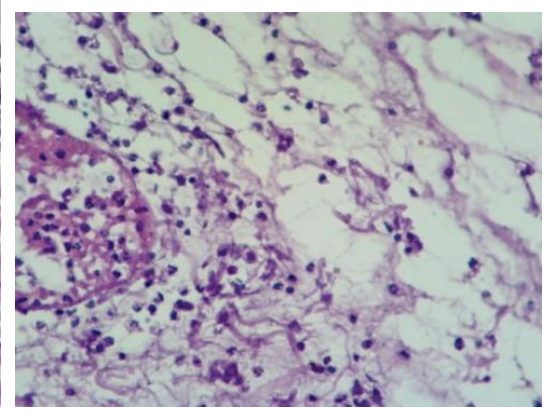

Subcutaneous

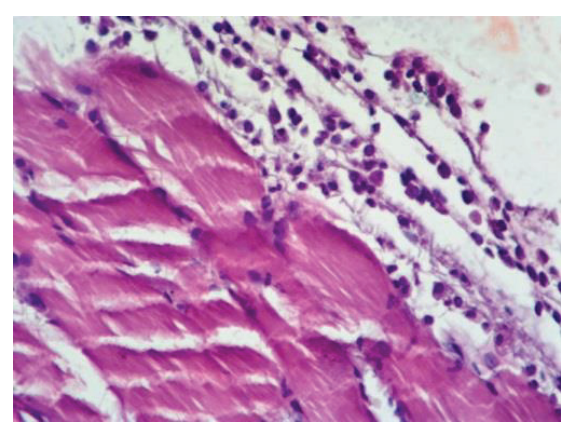

Muscle

Fig. 6 Skin, subcutaneous, and muscle tissue condition after SC injection of carrageenan weekly for 1 month and single IM injection of DSP solution at day 0 .

and its effect started from the first day of using it for acute inflammation as agreed with reported data. ${ }^{20}$

The samples were taken after 1 month from group 6 in which the animals injected with carrageenan SC injection weekly for 1 month and also injected with intramuscular DSP at 0 days only. The result showed a marked increase in the infiltration of inflamed cells (Lymphocytes and neutrophil) as shown in Fig. 6. There is no significant difference with the result obtained in group 3 (the rats were injected with carrageenan only weekly for 1 month) indicating that the use of intramuscular DSP which had no effect on the number of infiltrated inflamed cells after 1 month. This means that the anti-inflammatory effect of single IM DSP injection was diminished after 1 month because the half-life of DSP is 36-45 h. Hence, the single IM injection of DSP has no effect on chronic inflammation. ${ }^{21}$

The samples were taken after 1 day from group 7 in which the animals were injected with SC injection of carrageenan solution with the insertion of implantable film at the site of injection containing microencapsulated DSP. The result showed a marked significant decrease in the inflammatory cells in all the tissues (skin, subcutaneous, and muscle tissue) in comparison to the results obtained from group 2 as shown in Fig. 7. This indicated that the rapid release of the drug from the implantable film had significant effect on the acute inflammation and much better effect than the IM injection of pure DSP solution that was observed in group 5 .

The samples taken after 1 month from group 8 in which the animals in this group were injected with SC injection of carrageenan weekly with the insertion of single implantable film at the site of injection at zero time. The result showed significant marked decrease in the inflammatory cells in all the tissues (skin, subcutaneous, and muscle tissue) in comparison to group 3 as shown in Fig. 8 and also significantly different from the result obtained from group 6 (where IM injection of pure DSP given at the site of injection of single SC carrageenan injection). This indicated the potent anti-inflammatory action of the implanted film on the chronic inflammation after 1 month in comparison to IM injection of pure DSP in group 6 which had no effect on the chronic inflammation after 1 month. The follow up of the anti- inflammatory effect of the implanted film was continued every month for 5 months and the results showed that no sign of inflammation observed in all skin, subcutaneous, and muscle tissues and similar to healthy tissues indicating that the amount of DSP released from the implanted film was effective for 5 months (as shown in Fig. 9). The overall results proved that the prepared implantable film had immediate anti-inflammatory effect upon insertion at the inflamed tissue and its action continued effectively for 5 months. These results were highly correlated with in vitro drug release study, ${ }^{13}$ which showed initial immediate release $(22.05 \%)$ of the total amount of DSP in the film within the first $6 \mathrm{~h}$ and the release continued for 5 months in which $(86.18 \%)$ of the total incorporated DSP was released after 5 months. Therefore, it is suggested to be implanted after surgery of joint replacement or any other surgery site to be used as alternative to the applied regimen of giving IM injection of the commercially available $10 \mathrm{mg}$ dexamethasone intraoperatively and a second $10 \mathrm{mg}$ within 24 -h post-operatively to reduce edema and pain followed by oral dexamethasone 0.5 mg daily till complete recovery which may last for 5 months or more, ${ }^{22}$ which subject the patients to the serious systemic

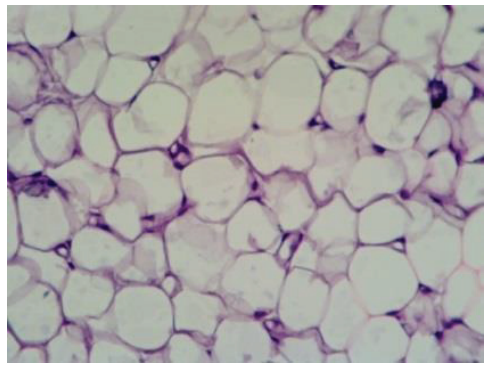

Skin

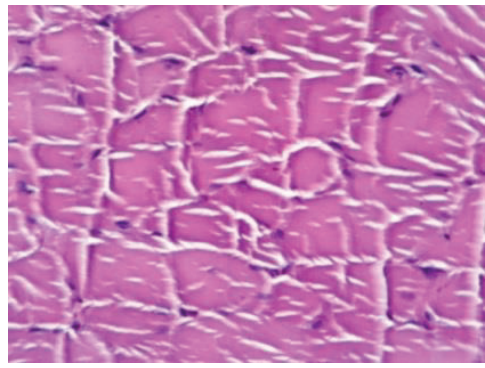

Subcutaneous

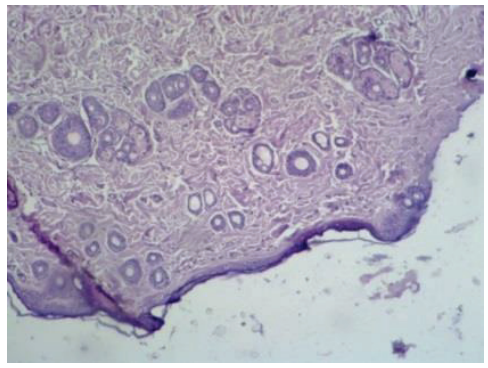

Muscle

Fig. 7 Skin, subcutaneous and muscle tissue condition after 1 day of SC injection of carrageenan and insertion of the prepared film at the same site of injection. 


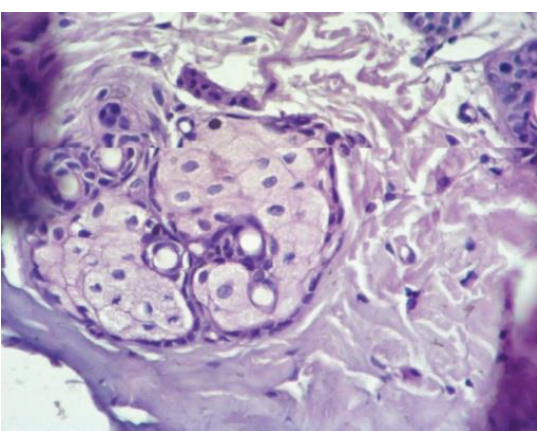

Skin

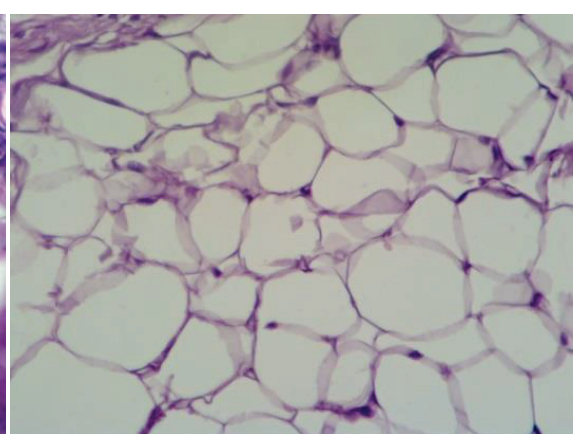

Subcutaneous

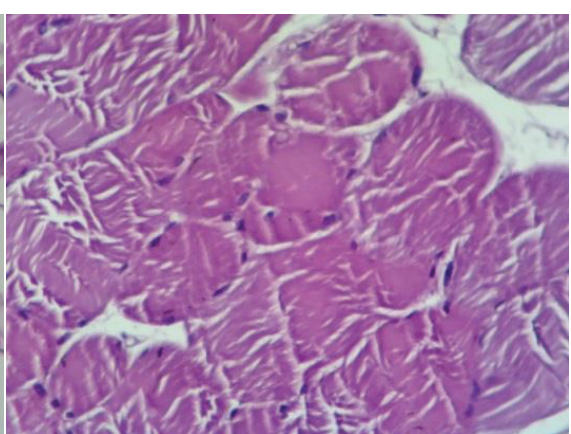

Muscle

Fig. 8 Skin, subcutaneous, and muscle tissue after weekly SC injection of carrageenan solution for 1 month and insertion of single prepared film at the site of injection at zero time.
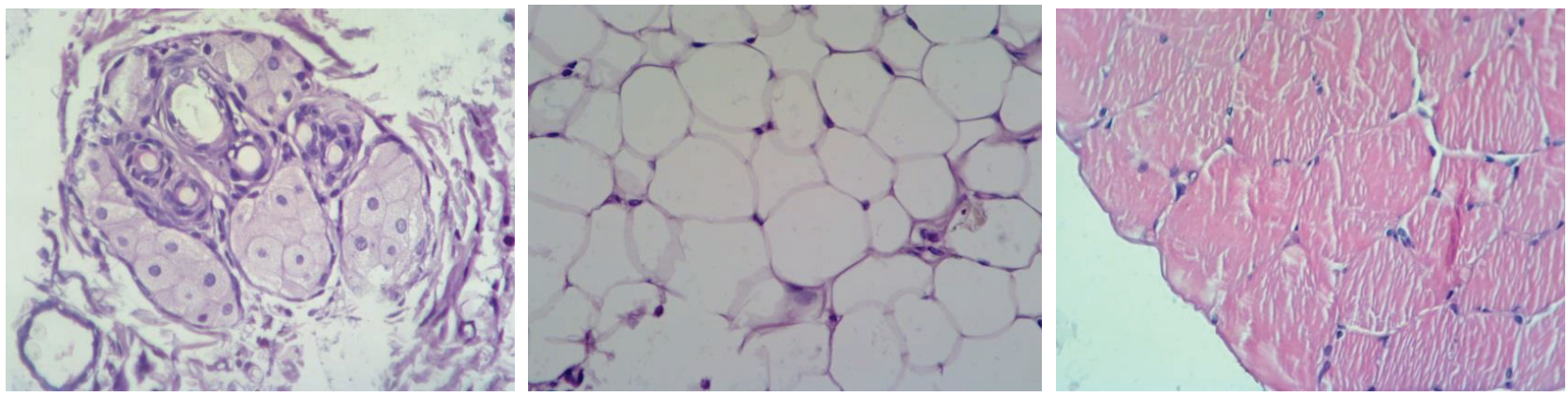

Fig. 9 Skin, subcutaneous, and muscle tissue after weekly SC injection of carrageenan solution for 5 months and insertion of single prepared film at the site of injection at zero time.

effects. The application of the implanted film therapy may reduce the rejection of the body to the replaced joint or other replacement surgery and improves patient compliance.

\section{Conclusion}

DSP was previously microencapsulated by PLGA polymer, then the microcapsules were incorporated on PVA-based film to produce implantable film containing $17.6 \mathrm{mg}$ DSP (as microcapsule equivalent to $16 \mathrm{mg}$ dexamethasone) and 4.4 mg pure DSP (uncoated drug equivalent to $4 \mathrm{mg}$ dexamethasone). The in vivo evaluation showed that the prepared film is success fully implanted and had remarkable effectiveness against acute inflammation (within day 1 of insertion) and continued effective for 5 months against chronic inflammation and the results were in a good agreement with in vitro data, which make the prepared film preferable to be used as alternative to dexamethasone conventional regimen treatment to reduce frequent doses and their side effects and improve patient compliance

\section{References}

1. Serhan C N, Savill J . Resolution of inflammation: The beginning programs the end. Nat Immunol. 2005;6(12):1191-1197.

2. Ahmed, Afsar. An overview of inflammation: Mechanism and consequences. Front Biol. 2011:6(4):274-281.

3. Hotaling, N.A.; Tang, L.; Irvine, D.J.; Babensee, J.E. Biomaterial strategies for immunomodulation. Annu Rev Biomed Eng. 2015:17:1-16.

4. Balint, R.; Cassidy, N.J.; Cartmell, S.H. Conductive polymers: Towards a smart biomaterial for tissue engineering. Acta Biomater. 2014;10:2341-2353.

5. Latour, R.A. Biomaterials: Protein-surface interactions. Encycl Biomater Biomed Eng. 2005;28:1-15.

7. Bozic, K. J. et al. The epidemiology of bearing surface usage in total hip arthroplasty in the United States. J Bone Joint Surg Am. 2009;91:1614-1620.

8. Goodman, S. B. Wear particles, periprosthetic osteolysis and the immune system. Biomaterials 2007;28:5044-5048.

9. Purdue, P. E., Koulouvaris, P., Potter, H. G., Nestor, B. J. \& Sculco, T. P. The cellular and molecular biology of periprosthetic osteolysis. Clin Orthop Relat Res. 2007:454:251-261

10. Yue, C. Perioperative systemic steroid for rapid recovery in total knee and hip arthroplasty: A systematic review and meta-analysis of randomized trials. J Orthop Surg Res. 2017;12-100
11. Bradford R., A.G. White, L.M. Scarpati, G. Wan, Long-term systemic corticosteroid exposure: A systematic literature review. Elsevier, 2017:39(11):2216-2229

12. Jeffrey R. Backes, Jared C. Bentley, Joel R. Politi, Bryan T. Chambers. Dexamethasone reduces length of hospitalization and improves postoperative pain and nausea after total joint arthroplasty a prospective, randomized controlled trial. J Arthropl. 2013;1:11-17.

13. Mohammed W., Nidhal K. Maraie. Preparation and in-vitro evaluation of implantable film containing microencapsulated sustained release dexamethasone sodium phosphate. Int J Pharmaceut Res. 2019;11(4).

14. Justin C. Earp, Nancy A. Pyszczynski, Diana S. Molano, and William J. Jusko. Pharmacokinetics of dexamethasone in a rat model of rheumatoid arthritis. Biopharm Drug Dispos J. 2008:29(6):366-372.

15. Chan JK. The wonderful colors of the hematoxylin-eosin stain in diagnostic surgical pathology. Int J Surg Pathol. 2014;22(1):12-32.

16. Chan JK. The wonderful colors of the hematoxylin-eosin stain in diagnostic surgical pathology. Int J Surg Pathol. 2014;22(1):12-32.

17. Butterfield TA, Best TM, Merrick MA. The dual roles of neutrophils and macrophages in inflammation: A critical balance between tissue damage and repair. J Athletic Train. 2006;41(4):457-465. 
18. Atul R. Chopade, Fahim J. Sayyad, Nilofar S. Naikwade. Pharmacological characterization of carrageenan induced heat muscle hyperalgesia in rats using non-selective, preferential and selective COX-2 inhibitors. Pharmacol Rep. 2014:66:353-362.

19. J.S.Lindholt. Chronic inflammation, immune response, and infection in abdominal aortic aneurysms. Eur J Vasc Endovasc Surg. 2006;31(5):453-463.

20. Yeo Y, Park K. Control of encapsulation efficiency and initial burst in polymeric microparticle systems. Arch Pharm
21. Ram C Dhakar, S. D. From formulation variables to drug entrapment efficiency of microspheres: A technical review. J Drug Deliv Therap. 2012;2(6):128-133.

22. Jeffrey R. Backes, Jared C. Bentley, Joel R. Politi, Bryan T. Chambers. Dexamethasone reduces length of hospitalization and improves postoperative pain and nausea after total joint arthroplasty a prospective, randomized controlled trial. J Arthropl. 2013;1:11-17.

This work is licensed under a Creative Commons Attribution-NonCommercial 3.0 Unported License which allows users to read, copy, distribute and make derivative works for non-commercial purposes from the material, as long as the author of the original work is cited properly. 